\title{
Frequência de escovação e fatores associados em adolescentes: novas abordagens
}

\author{
- Jaqueline Montoril Sampaio Mota Pós-graduação no Departamento de Odontologia Social, Faculdade de \\ Odontologia, Universidade de São Paulo, São Paulo, Brasil • Paulo Roberto da Silva Pós-graduação no Departamento \\ de Odontologia Social, Faculdade de Odontologia, Universidade de São Paulo, São Paulo, SP, Brasil • Maria Gabriela \\ Haye Biazevic Departamento de Odontologia Social, Faculdade de Odontologia, Universidade de São Paulo, São \\ Paulo, SP, Brasil - Edgard Michel-Crosato Departamento de Odontologia Social, Faculdade de Odontologia, \\ Universidade de São Paulo, São Paulo, SP, Brasil
}

RESUMO | Objetivos: verificar a relação entre frequência de escovação e fatores associados utilizando novas perspectivas de análise de dados em saúde. Materiais e métodos: foi realizado um estudo transversal, com dados coletados da Pesquisa Nacional de Saúde do Escolar 2015 (PeNSE), foi utilizada a regressão de Poisson Multinível e Multivariada e técnica de mineração de dados utilizando a técnica de Árvore de Classificação. Resultados: participaram do estudo 10.231 escolares, sendo 51,3\% dos escolares do gênero feminino com idades entre 13 a 18 anos. $\mathrm{Na}$ análise multivariada e multinível foi verificada associação entre frequência de escovação e não morar em capitais ( $\mathrm{RP}=1.03)$; ser do gênero feminino $(\mathrm{RP}=1.06)$; ser branco ( $\mathrm{RP}=1.02)$; idade; e morar em casas com até 4 pessoas ( $\mathrm{RP}=1.03)$. Na análise de árvore de classificação o gênero foi a variável com maior peso de discriminação. Conclusões: vários fatores foram associados à frequência de escovação e espera-se que os dados subsidiem políticas públicas sobre a questão.

DESCRITORES | Saúde Bucal; Prevenção; Autocuidado; Epidemiologia; Big Data; Metodologia.

ABSTRACT | Frequency of toothbrushing and associated factors in adolescents: new approaches - Objectives: To verify the relationship between tooth brushing frequency and associated factors using new perspectives of health data analysis. Materials and methods: A cross-sectional study was conducted with data collected from the Brazilian National School Health Survey 2015 (PeNSE), we used multilevel and multivariate Poisson regression and classification tree analysis data mining technique. Results: 10,231 students participated in the study, of which $51.3 \%$ were female students aged 13 to 18 years. In the multivariate and multilevel analysis, association between frequency of brushing and non-living in a state capital ( $\mathrm{PR}=1.03$ ) was verified; being female $P R=1.06$ ); race $P R=1.02$ ); age; and live in houses with up to 4 people $P R=1.03$ ). In the classification tree analysis, gender was the variable with the highest weight of discrimination. Conclusions: several factors were associated with the tooth brushing and it is expected that the data will subsidize public policies on the issue.

DESCRIPTORS | Oral Health; Prevention; Self-Care; Epidemiology; Big Data; Methodology.

AUTOR CORRESPONDENTE

Edgard Michel-Crosato Faculdade de Odontologia da Universidade de São Paulo • Avenida Professor Lineu Prestes, 2227 São Paulo, SP, Brasil •05508-000 E-mail: michelcrosato@usp.br

- Received Sep 27, 2018 • Accept Nov 22, 2018

- Dol http://dx.doi.org/10.11606/issn.2357-8041.clrd.2019.150619 


\section{INTRODUÇÃO}

Nos países em desenvolvimento, principalmente, a cárie dentária se apresenta ainda como um dos principais problemas de saúde bucal da população, com enfoque em localidades menos favorecidas, que apresentam alto consumo de açúcar, baixa exposição a fluoretos e reduzida frequência de autocuidados em saúde bucal. ${ }^{1,2}$

Embora a prevalência de cárie esteja em declínio, principalmente aos 12 anos de idade, no período da adolescência, existe um incremento substancial dessa prevalência. ${ }^{3,4}$

A adolescência, como fase do ciclo de vida, apresenta-se como a transição da infância para a vida adulta, trazendo importantes transformações sociais e biológicas aos indivíduos. Essa fase, é marcada por crescente autonomia e independência em relação à família e experimentação de novos comportamentos.,56

A escovação dentária é uma prática de autocuidado importante para a manutenção de uma saúde bucal adequada.7 No período da adolescência, essa prática de autocuidado pode se modificar por fatores externos, e é importante conhecermos os motivos que podem estar associadas a uma maior, ou menor, frequência de escovação. ${ }^{8}$

Inquéritos populacionais têm sido muito utilizados para o monitoramento de saúde, várias pesquisas têm se desenvolvido no Brasil, como as pesquisas nacionais da saúde do adolescente. A Pesquisa Nacional de Saúde do Escolar (PeNSE), que se iniciou no Brasil em 2009, teve sua segunda versão em 2012 e em 2015 foi realizada a terceira coleta. ${ }^{9}$ Todas elas apresentam dados de saúde bucal e com os dados de 2009 foi realizado estudo para verificar a frequência de escovação e fatores socioeconômicos. ${ }^{6}$ Para reforçar esse tipo de estudo, Gil et al. ${ }^{10}$ afirmam que a frequência autorreferida de escovação pode ser utilizada como medida indireta para índices clínicos de higiene bucal, o que facilita a coleta de dados em estudos epidemiológicos de saúde bucal em adolescentes. Outro fator relevante é que grandes inquéritos populacionais propiciam outros tipos de análise dos dados, e não apenas as estatísticas tradicionais. O uso de técnicas de grandes bancos, mineração de dados e aprendizagem de máquinas podem ser utilizados, somados aos procedimentos estatísticos tradicionais. ${ }^{11}$

Nesse contexto, o objetivo deste estudo foi verificar a relação entre frequência da escovação dental e fatores associados, utilizando novas perspectivas de análise de dados em saúde.

\section{MÉTODO}

\section{Delineamento do estudo}

Foi realizado um estudo transversal, tipo inquérito, utilizando dados da Pesquisa Nacional de Saúde do Escolar 2015 (PeNSE).

\section{População de estudo}

A amostra foi composta por escolares do nono ano do ensino fundamental devidamente matriculados, no ano letivo de 2015, e frequentando regularmente escolas públicas e privadas situadas nas zonas urbanas e rurais de todo o Brasil.

O tamanho da amostra de cada estrato geográfico foi calculado para fornecer estimativas de proporções (ou prevalências) de algumas características de interesse, com um erro amostral máximo aproximado de $3 \%$, em valores absolutos, e nível de confiança de 95\%.

$$
n=\text { epa. } n_{A A S}=e p a \cdot \frac{1,96^{2}}{4 d^{2}+1,96^{2} / N}
$$

Onde:

$n$ é o tamanho da amostra de alunos em um determinado estrato geográfico;

$n_{A A S}$ é o tamanho de uma amostra aleatória simples equivalente;

$d$ é a margem de erro esperada;

$N$ é o total de alunos no cadastro nesse estrato geográfico;

epa é uma estimativa do efeito de conglomeração. 


\section{Procedimentos}

Os dados foram coletados por meio de aparelhos smartphones, nos quais foram inseridos os questionários estruturados, autoaplicáveis, com módulos temáticos que variavam em número de perguntas. A coleta foi desenvolvida por agentes do Instituto Brasileiro de Geografia e Estatística (IBGE), devidamente treinados para este fim, e os dados foram coletados nas escolas, durante o horário de aulas dos alunos. Maiores detalhes sobre a metodologia podem ser obtidos em publicações específicas. ${ }^{9}$

\section{Análise de dados}

Os dados da PeNSE 2015 foram exportados para uma planilha de dados Excel e os dados foram trabalhados para a limpeza dos mesmos e para a preparação do banco de dados. Após essa etapa, os dados foram analisados no Pacote Estatístico STATA 14. Foram realizadas análise de regressão bivariada e permaneceram no modelo apenas as variáveis que apresentaram razão de prevalência com significância menor ou igual a 0.20. A variável de desfecho foi considerada de forma dicotômica, escovar os dentes até duas vezes ao dia e escovar três vezes ou mais. Depois foram realizadas regressões de Poisson Multivariada e Multivariada e Multinível considerando os estados brasileiros. Para finalizar, o banco foi trabalhado nos programas R e Python e foram utilizadas a técnica de mineração de dados e aprendizado e máquina, utilizando a técnica de árvore de decisão. Para essa análise foi feito o teste de acurácia da área da curva ROC, para o grupo treino (70\%) da amostra e (30\%) para o grupo teste. O nível de significância foi de $5 \%$.

\section{Ética}

A PeNSE 2015 foi aprovada pela Comissão Nacional de Ética em Pesquisa (Conep), vinculada ao Conselho Nacional de Saúde (CNS), que regulamenta e aprova pesquisas em saúde envolvendo seres humanos, por meio do Parecer de número 1.006.467, de 30 de março de 2015.

\section{RESULTADOS}

Participaram do estudo 102.301 escolares matriculados no nono ano do ensino fundamental. A Tabela 1 apresenta o tamanho amostral esperado e coletado no estudo.

Em relação às principais variáveis de desfecho e exposição, 73,43\% dos escolares escovam o dente três ou mais vezes por dia; 51,71 eram do gênero feminino, 50,60\% tinham 14 anos de idade, 50,15\% moram em capitais (Tabela 2).

Buscando os fatores que podem estar associados com a frequência de escovação, foi realizada a Regressão de Poisson, aplicada apenas às variáveis que apresentaram razão de prevalência com significância menor ou igual a 0.20 e que continuaram no modelo de verificação de risco. As variáveis que verificaram possíveis associações são: não morar em capitais ( $\mathrm{RP}=1.03)$; ser do gênero feminino ( $\mathrm{RP}=1.06)$; ser branco $(\mathrm{RP}=1.04)$; idade; $\mathrm{e}$ morar em casas com até quatro pessoas $(\mathrm{RP}=1.04)$ (Tabela 3). Esses resultados apontam que as variáveis citadas estão presentes em pessoas que escovam mais a boca. Dessa forma, participantes que moram nas capitais, do gênero masculino, de cor de pele não branca e que moram com mais de quatro pessoas apresentam um risco maior de escovarem menos frequentemente a boca.

Tabela 1 | Tamanho amostral dos escolares esperado e coletado, Brasil 2015.

\begin{tabular}{c|c|c|c|c|c|c|c|c|} 
Unid. & \multicolumn{3}{|c|}{ Esperado } & \multicolumn{3}{c}{ Coletado } \\
& Escolas & Turmas & Alunos & Escolas & Turmas & Alunos & Respondentes \\
\hline Brasil & 3.160 & 4.418 & 128.027 & 3.040 & 4.159 & 124.227 & 102.301 \\
\hline
\end{tabular}


Na análise multivariada e multinível, foram verificados os mesmos preditores de risco da análise multivariada, com pequenas variações na razão de prevalência. Os resultados mostraram diferenças entra as regiões do país para a frequência de escovação, o que indicou a utilização da análise multinível (Tabela 4). Também foi verificado o mesmo critério para a utilização de diferentes padrões na frequência de escovação. Foram verificadas associações entre a frequência de escovação e não morar em capitais ( $\mathrm{RP}=1.03)$; ser do gênero feminino ( $\mathrm{RP}=1.06)$; ser branco $(\mathrm{RP}=1.02)$; idade; e morar em casas com até quatro pessoas $(\mathrm{RP}=1.03)$.
Utilizando a técnica de mineração de dados, a melhor variável de discriminação para a maior frequência de escovação foi o gênero. Essa técnica melhora a visualização dos dados. O primeiro nó de avaliação foi o gênero, pois 75,4\% das participantes do gênero feminino escovam seus dentes três ou mais vezes por dia, enquanto para o gênero masculino esse grupo foi de $71,3 \%$. Para o gênero feminino, o segundo nó de avaliação foi não morar em capital; para o gênero masculino, foi a cor da pele, no caso a cor de pele branca. Dessa forma podemos analisar os grupos de risco integrados por níveis hierárquicos (Figura 1). A acurácia para a curva ROC para a fase treino foi de 0.87 e para a fase teste foi de 0.79 .

Tabela 2 | Distribuição dos escolares segundo os fatores de risco. Brasil, 2015.

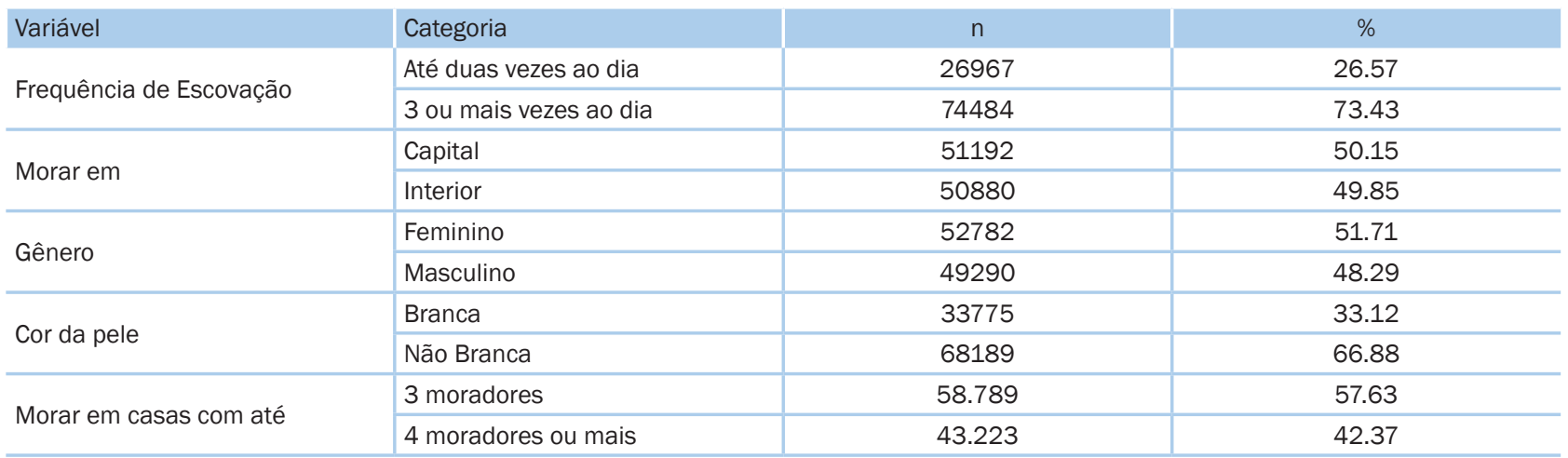

Tabela 3 | Risco relativo multivariado dos escolares em relação aos fatores de risco. Brasil, 2015.

\begin{tabular}{l|l|l|l|l|r|r}
\hline Variáveis & RP* & SE** & Z*** & $P_{* * * *}$ & IC 95\%***** \\
\hline Não capital & 1.03 & 0.008 & 4.51 & 0.000 & $1.02-1.05$ \\
\hline Feminino & 1.06 & 0.008 & 7.39 & 0.000 & $1.04-1.07$ \\
\hline Brancos & 1.04 & 0.008 & 4.90 & 0.000 & $1.02-1.06$ \\
\hline Menos de 4 pessoas & 1.04 & 0.008 & 5.44 & 0.000 & $1.02-1.06$ \\
\hline
\end{tabular}

* Razão de prevalência; ** Erro padrão; *** Estatística Z; **** Valor de p; ***** Intervalo de confiança 95\%.

Tabela 4 | Risco relativo multivariado e multinível dos escolares em relação aos fatores de risco. Brasil, 2015.

\begin{tabular}{|c|c|c|c|c|c|}
\hline Variáveis & $\mathrm{RP} *$ & $\mathrm{SE} * *$ & $Z^{* * *}$ & $\mathrm{P} * * * *$ & IC $95 \% * * * * *$ \\
\hline Não capital & 1.03 & 0.008 & 4.19 & 0.000 & $1.02-1.05$ \\
\hline Feminino & 1.06 & 0.008 & 7.32 & 0.000 & $1.04-1.07$ \\
\hline Brancos & 1.02 & 0.008 & 3.10 & 0.002 & $1.01-1.04$ \\
\hline Menos de 4 pessoas & 1.03 & 0.008 & 4.22 & 0.000 & $1.02-1.05$ \\
\hline \multicolumn{6}{|l|}{2 estágio } \\
\hline Microregião & 2.71 & - & - & 0.000 & - \\
\hline
\end{tabular}

* Eazão de prevalência; ** Erro padrão; *** Estatística Z; **** Valor de p; ***** Intervalo de confiança. 


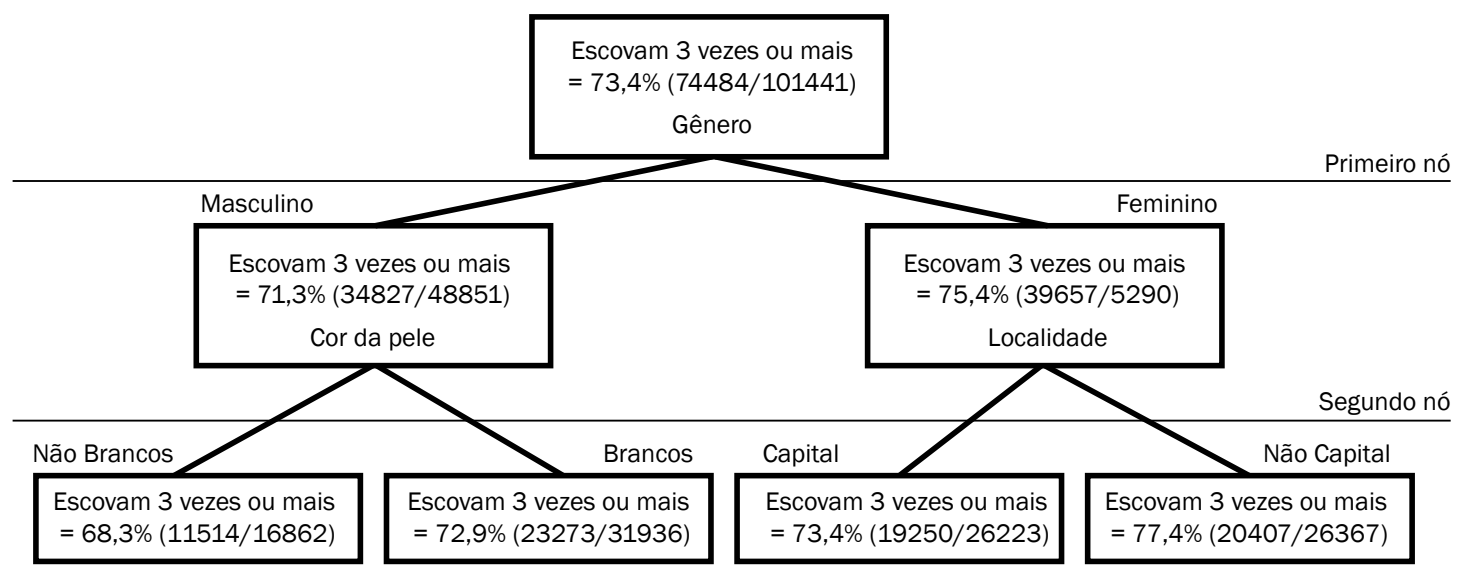

Terceiro nó

Figura 1 | Análise de árvore de classificação para a frequência de escovação dentária dos escolares. Brasil 2015.

\section{DISCUSSÃO}

É indiscutível que várias políticas nacionais priorizam a população de jovens para a promoção da saúde. Nesse contexto, a escola constitui um espaço coletivo privilegiado. ${ }^{12}$ No presente estudo, a escolha do nono ano do ensino fundamental teve como justificativa o mínimo de escolarização e a capacidade para responder ao questionário autoaplicável corretamente..$^{13,14}$

Uma limitação desse estudo é que a PeNSE 2015 utiliza amostras apenas de estudantes, e o acesso à escola para adolescentes de 15 a 19 anos é de aproximadamente $88 \%$ no Brasil. ${ }^{15}$ Essa estratégia metodológica pode resultar em um viés de informação, com superestimação de comportamentos de promoção da saúde e subestimação de comportamentos de risco para a saúde. ${ }^{16}$

Embora ainda existam discussões na comunidade científica sobre a real efetividade da frequência da escovação dentária para a prevenção da cárie, essa prática é de fundamental importância para o cuidado bucal e para a promoção de saúde bucal. ${ }^{14}$

Dados do último levantamento epidemiológico brasileiro, de 2010, ${ }^{17}$ demonstram que o número médio de dentes cariados, perdidos e obturados
(CPO-D) aos 12 anos era de 2,07 e na faixa etária de 15 a 19 era de 4,25 . Isso demostra o grande aumento da prevalência de cárie que ocorre nesse período, ${ }^{18}$ o que confirma a grande importância da promoção de saúde bucal e de autocuidados em saúde bucal nessa faixa etária.

Diferente de outros estudos, em relação à frequência de escovação, utilizamos o corte dicotômico entre escovar os dentes três vezes ou mais e não duas vezes ou mais. Essa decisão foi tomada mediante o resultado encontrado no estudo de Vettore et al., ${ }^{6}$ em que 96,00\% dos escolares escovavam os dentes mais de duas vezes por dia. No nosso estudo foi verificado a mesma tendência com cerca de 92,00\% de participantes nessa condição. Utilizando como fator de corte escovar os dentes três vezes ou mais, foi verificada a prevalência de $73,43 \%$. Com esse método, discriminamos melhor a amostra para a análise multivariada. ${ }^{6,19}$

Em relação aos fatores de risco, a literatura mostra estar associada ao sexo feminino, ${ }^{6,20,21}$ à melhor condição socioeconômica, ${ }^{6,20}$ à escolaridade ${ }^{6}$ e ao tipo de serviço utilizado. ${ }^{22}$ No presente estudo foi encontrado padrão similar, pois a frequência de escovação foi mediada pelos fatores: não morar em capitais; ser do gênero feminino; ser branco; idade; e 
morar em casas com até quatro pessoas. Na técnica de árvore de classificação, o fator mais importante foi o gênero. Para o masculino, o fator de maior destaque foi ter a cor da pele branca e para o feminino, foi morar fora de capital. Embora o fator de acurácia não tenha sido alto, ele variou pouco entre o treino e o teste, demostrando uma validade externa. Portanto, essa análise pode ser extrapolada para outros bancos de dados. Outra característica da árvore de classificação é que apresenta os nós de avaliação e a hierarquia das variáveis mais importantes para serem avaliadas em uma nova proposta de política pública para o tema, variando a abordagem entre os gêneros.

As técnicas de mineração de dados, grandes bancos e aprendizagem de máquinas podem ser aplicadas para o estudo de ações de promoção e prevenção da saúde, de modo a melhorar a organização dos serviços de saúde. As limitações dessas técnicas são a maior dificuldade da construção de bancos de dados, muitas vezes baseados em informações incompletas e em informações contraditórias. Mas vale ressaltar que esses métodos são bastante promissores e podem ajudar na tomada de decisão e elaboração de ações para a melhoria da qualidade de vida da população. ${ }^{11,23}$

\section{CONCLUSÃO}

A frequência de escovação foi mediada pelos fatores: não morar em capitais; ser do gênero feminino; ser branco; idade; e morar em casas com até quatro pessoas. Espera-se que os dados subsidiem políticas públicas sobre a questão, uma vez que oferecem indicadores que podem auxiliar no delineamento de estratégias de enfrentamento, principalmente no que se refere a novas abordagens metodológicas.

\section{AGRADECIMENTOS}

A todas as pessoas que participaram da PeNSE 2015 .

\section{REFERÊNCIAS}

1. Pitts N, Amaechi B, Niederman R, Acevedo AM, Vianna $\mathrm{R}$, Ganss $\mathrm{C}$ et al. Global oral health inequalities: dental caries task group: research agenda. Adv Dent Res. 2011 May;23(2):211-20. doi:10.1177/0022034511402016.

2. Silva CAT, Rebelo Vieira JM, Rebelo MA, Vettore MV. The association between participation of adolescents in community groups and dental caries in a deprived area in Brazil. Caries Res. 2015;49(5):540-7. doi:10.1159/000438726.

3. Lopes MC, Silva PR, Biazevic MGH, Rebelo MAB, Crosato EM. Need for dental treatment due to caries in 15-19-year-old students of Manaus, AM, Brazil, and associated factors. Pesq Bras Odontoped Clin Integ. 2012;12(1):83-8. doi:10.4034/pboci.v12i1.1158.

4. Silveira MF, Freire RS, Nepomuceno MO, Martins AMEBL, Marcopito LF. Tooth decay and associated factors among adolescents in the north of the State of Minas Gerais, Brazil: a hierarchical analysis. Ciência \& Saúde Coletiva. 2015;20(11):335164. doi:10.1590/1413-812320152011.12262014.

5. Ruzany MH, Szwarcwald CL. Oportunidades perdidas de atenção integral ao adolescente: resultado de estudo piloto. Rev Adolesc LatinoAme. 2000;2(1):26-35.

6. Vettore MV, Moysés SJ, Sardinha LMV, Iser BPM. Condição socioeconômica, frequência de escovação dentária e comportamentos em saúde em adolescentes brasileiros: uma análise a partir da Pesquisa Nacional de Saúde do Escolar (PeNSE). Cad Saúde Pública. 2012;28:S101-13. doi: 10.1590/So102311X2012001300011.

7. Poklepovic T, Worthington HV, Johnson TM, Sambunjak D, Imai P, Clarkson JE, et al. Interdental brushing for the prevention and control of periodontal diseases and dental caries in adults. Cochrane Database Syst Rev. 2013;12:CDoo9857. doi: 10.1002/14651858.CDoo9857.pub2.

8. Sadeghipour M, Khoshnevisan MH, Jafari A, Shariatpanahi SP. Friendship network and dental brushing behavior among middle school students: An agent based modeling approach. PLoS ONE. 2017;12(1): e0169236. doi: 10.1371/journal. pone.0169236.

9. IBGE. Pesquisa nacional de saúde do escolar 2015. Rio de Janeiro: IBGE; 2016. 132 p.

10. Gil GS, Morikava FS, Santin GC, Pintarelli TP, Fraiz FC, Ferreira FM. Reliability of self-reported toothbrushing frequency as an indicator for the assessment of oral hygiene in epidemiological research on caries in adolescents: a cross-sectional study. BMC Med Res Methodol. 2015;15:14. doi: 10.1186/s12874-015-0002-5. 
11. Chiavegatto Filho ADP. Uso de big data em saúde no Brasil: perspectivas para um futuro próximo. Epidemiol. Serv. Saúde. 2015;24(2):325-32. doi: 10.5123/S1679-49742015000200015.

12. Moysés ST, Moysés SJ, Watt RG, Sheiham A. Associations between health promoting schools' policies on some indicators of oral health in Brazil. Health Promot Int. 2003;18(3):20918. doi: 10.1093/heapro/dag/o16.

13. Oliveira WA. Silva MAI, Mello FCM, Porto DL, Yoshinaga ACM, Malta DC. Causas do bullying: resultados da Pesquisa Nacional do Escolar. Rev Latino-Am Enfermagem. 2016;23(2): 275-82. doi: 10.1590/0104-1169.0022.2552.

14. Kumar S, Tadakamadla J, Johnson NW. Effect of toothbrushing frequency on incidence and increment of dental caries: A systematic review and meta-analysis. J Dent Res. 2016 Oct;95(11):1230-6. doi:10.1177/0022034516655315.

15. Pesquisa Nacional por Amostra de Domicílio. Síntese de indicadores 2011. Rio de Janeiro: IBGE; 2012. 282 p.

16. Azeredo CM, Levy RB, Peres MF, Menezes PR, Araya R. Patterns of health-related behaviours among adolescents: a cross-sectional study based on the National Survey of School Health Brazil 2012. BMJ Open. 2016;6(11):e011571. doi:10.1136/bmjopen-2016-011571.

17. Ministério da Saúde. Pesquisa Nacional de Saúde Bucal: resultados principais. Brasília, 2012: Ministério da Saúde; 2012. 118 p.
18. Ely HC, Abegg C; Rosa AR, Pattussi MP. Redução da cárie dentária em adolescentes: distribuição temporal e espacial em 36 municípios do Sul do Brasil 2003 e 2011. Epidemiol Serv Saúde. 2014;23(3):421-34. doi: 10.5123/S167949742014000300005 .

19. Maes L, Vereecken C, Vanobbergen J, Honkala S. Tooth brushing and social characteristics of families in 32 countries. Int Dent J. 2006;56(3):159-67. doi: 10.1111/j. 1875-595X.2006.tbooo89.x.

20. Abegg C. Hábitos de higiene bucal de adultos porto-alegrenses. Rev. Saúde Pública. 1997;31(6):586-93. doi: 10.1590/ Soo34-89101997000700007.

21. Freddo SL, Aerts DNGC, Abegg C, Davoglio R, Vieira PC, Monteiro L. Hábitos de higiene bucal e utilização de serviços odontológicos em escolares de uma cidade da Região Sul do Brasil. Cad. Saúde Pública. 2008;24(9):1991-200o. doi: 10.1590/S0102-311X2008000900005.

22. Davoglio RS, Aerts DRGC, Abegg C. Freddo SL, Monteiro L. Fatores associados a hábitos de saúde bucal e utilização de serviços odontológicos entre adolescente. Cad Saúde Pública. 2009;25(3):655-67. doi: 10.1590/So102311X2009000300020.

23. Souza AP, Zaia JE. O uso do data mining na promoção de saúde: uma revisão sistemática da literatura. Atas de Saúde Ambiental 2015;3(1):12-21. 